\title{
EDITORIAL \\ To pace or not to pace: that is the question
}

\author{
Atul Verma, MD, ${ }^{1}$ Subodh Verma, MD, PhD, ${ }^{2}$ Mohammed Al-Omran, MD, MSc, ${ }^{1}$ and \\ James T. Rutka, MD, PhD³
}

${ }^{1}$ Division of Vascular Surgery, ${ }^{2}$ Division of Cardiac Surgery, and ${ }^{3}$ Division of Neurosurgery, Department of Surgery, University of Toronto, Ontario, Canada

$\mathrm{K}$ ONCZALLA and colleagues report on 20 patients undergoing clipping of complex, unruptured intracranial aneurysms (UIAs). ${ }^{4}$ The study utilized the technique of rapid ventricular pacing (RVP) to reduce the mean arterial pressure (MAP) to facilitate aneurysm preparation and clip application. Of the 20 patients studied, 16 underwent RVP at a mean rate of $173 \mathrm{bpm}$ for an average of 60 seconds to achieve a reduction in MAP to $35-55 \mathrm{~mm} \mathrm{Hg}$. In 15 of these patients, RVP allowed for successful aneurysm clipping, and in 1 patient, RVP failed due to pacemaker electrode dislocation.

In the field of cardiac electrophysiology (study of cardiac arrhythmias) and percutaneous aortic valve replacement, ventricular pacing is common. Temporary pacing electrodes are inserted routinely in patients with bradyarrhythmias who require temporary pacing support. They are also inserted in almost every patient undergoing cardiac electrophysiology studies to diagnose and treat the cause of arrhythmias. Catheter-based electrodes are typically inserted through central venous access (femoral, subclavian, internal jugular) into the right ventricular apex with a very low risk of cardiac perforation or tamponade (much less than 1\%). The ventricle can then be paced using an external pulse generator such as a temporary pacing box or an external stimulator. RVP up to $300 \mathrm{bpm}$ is often used to induce ventricular arrhythmias in patients with structural heart disease or to test the functioning of implantable cardioverter defibrillators. When such arrhythmias are induced, they can either be terminated by overdrive pacing in the ventricle (pacing faster than the arrhythmia) or by external cardioversion/defibrillation. Mortality and morbidity associated with such procedures are very rare. ${ }^{6}$ Thus, the idea of using RVP for short durations during an invasive procedure is considered neither a foreign nor dangerous concept in cardiology.

RVP reduces the MAP by reducing diastolic ventricular filling time and also changing the activation sequence of ventricular contraction (from apex to base instead of the opposite), both of which contribute to a reduction in stroke volume. In fact, in cardiac electrophysiology, RVP is employed to optimize acquisition of 3D contrast images of cardiac chambers. Highly detailed 3D reconstructions of the left atrium, for example, can be obtained by injecting radiopaque contrast into the chamber during RVP to optimize chamber filling and minimize motion artifact. ${ }^{2} \mathrm{RVP}$ with rates of 140-200 bpm are also used routinely during transcatheter aortic valve replacements to decrease stroke volume and mean aortic pressure during balloon valvuloplasty of the aortic valve and deployment of the valve. ${ }^{3}$ This is even used in patients with depressed ventricular function or nonrevascularized coronary disease, although the duration of RVP is minimized in these cases.

In the report by Konczalla and colleagues, ${ }^{4}$ the most common complication reported was dislocation of the pacing electrode (3/16 patients, 19\%). Given that repositioning of the electrode is a simple thing to do, requiring only a few seconds, it is unfortunate that 2 patients had their procedures changed due to "failure" of the RVP technique when a simple electrode repositioning would have corrected the issue. The authors fortunately changed their protocol to allow intraprocedural electrode repositioning, and as they reported themselves, this took less than 20 seconds.

This is not to say that RVP does not carry any significant risk, and induction of arrhythmias is always a concern. Two (12.5\%) of 16 patients suffered induction of arrhythmias (1 ventricular fibrillation and 1 atrial fibrillation) in this study. Whereas atrial fibrillation is relatively benign, ventricular fibrillation is a significant complication, especially because all patients had no significant structural heart disease according to preoperative cardiac screening. In this case, RVP was continued for 2 minutes, which was 
certainly too long. While the authors suggest that safety was improved by limiting the RVP to 100 seconds, shorter bursts (45-60 seconds) separated by a few seconds of rest would be a better approach to minimize pro-arrhythmia.

The risks and benefits of RVP also must be weighed against other methods of induced hypotension. As the authors note, a bolus of adenosine can also achieve transient ventricular standstill with reduction in MAP through induction of high-grade atrioventricular block. However, high doses are often required (60-100 mg), and asystole may occur unpredictably (10-30 seconds after bolus). ${ }^{1}$ Hypotension may also linger for more than $80-100$ seconds, but shorter than the 5-10 minutes suggested by the authors. ${ }^{1}$ Adenosine also causes diaphragmatic contractions (hiccups) in about $10 \%$ of patients, which can cause a lot of movement and may require paralytic agents. ${ }^{1}$ The agent also induces atrial fibrillation in $10 \%-15 \%$ of patients. Adenosine is also quite expensive in most jurisdictions, which should not be overlooked. Other vasodilator agents for inducing hypotension (nitrates, hydralazine, etc.) are all longer acting and also have less predictable responses at various doses. RVP responses are much more predictable and cease once pacing is stopped.

Temporary clipping of vessels proximal to the UIA is also feasible, and has been used by many surgeons, but is not without substantial risk of both ischemic and thromboembolic injury. A recent analysis suggested that there is an absolute $11 \%$ increase in the incidence of new ischemic lesions on CT and 14\% absolute increase in the risk of postoperative vasospasm. ${ }^{7}$ Furthermore, the duration of temporary occlusion may be limited to $2-4$ minutes before an increased risk of cerebral infarction. ${ }^{5} \mathrm{RVP}$ may provide longer durations of hypotension by several shorter applications (45-60 seconds) separated by just a few seconds.

RVP is an old technique in cardiology but may have an important "new" place in UIA repair. Ultimately, clinical studies directly comparing RVP with alternative techniques are required to determine the best methods for inducing transient hypotension and definitively answering the question: to pace or not to pace.

https://thejns.org/doi/abs/10.3171/2017.1.JNS162923

\section{References}

1. Ector J, De Buck S, Nuyens D, Rossenbacker T, Huybrechts W, Gopal R, et al: Adenosine-induced ventricular asystole or rapid ventricular pacing to enhance three-dimensional rotational imaging during cardiac ablation procedures. Europace 11:751-762, 2009

2. Hilbert S, Dagres N, Hindricks G, Sommer P, Gaspar T, Rallidis LS, et al: Rapid ventricular pacing: a fast, reliable, and safe technique for optimization of image acquisition during rotational angiography for catheter ablation of atrial fibrillation. Heart Vessels 26:349-352, 2011

3. Hilling-Smith R, Cockburn J, Dooley M, Parker J, Newton A, Hill A, et al: Rapid pacing using the 0.035 -in. retrograde left ventricular support wire in 208 cases of transcatheter aortic valve implantation and balloon aortic valvuloplasty. Catheter Cardiovasc Interv 89:783-786, 2017

4. Konczalla J, Platz J, Fichtlscherer S, Mutlak H, Strouhal U, Seifert V: Rapid ventricular pacing for clip reconstruction of complex unruptured intracranial aneurysms: results of an interdisciplinary prospective trial. J Neurosurg [epub ahead of print August 18, 2017: DOI: 10.3171/2016.11.JNS161420]

5. Lavine SD, Masri LS, Levy ML, Giannotta SL: Temporary occlusion of the middle cerebral artery in intracranial aneurysm surgery: time limitation and advantage of brain protection. Neurosurg Focus 87(6):817-824, 1997

6. Phan K, Ha H, Kabunga P, Kilborn MJ, Toal E, Sy RW: Systematic review of defibrillation threshold testing at de novo implantation. Circ Arrhythm Electrophysiol 9:e003357, 2016

7. Woertgen C, Rothoerl RD, Albert R, Schebesch KM, Ullrich OW: Effects of temporary clipping during aneurysm surgery. Neurol Res 30:542-546, 2008

\section{Disclosures}

The authors report no conflict of interest.

\section{Response}

\section{Volker Seifert, MD, and Juergen Konczalla, MD, for the Interdisciplinary Frankfurt RVP Research Team}

\author{
Department of Neurosurgery, University Hospital Frankfurt, Goethe \\ University, Frankfurt, Germany
}

We sincerely appreciate the thoughtful editorial by Verma and colleagues regarding our paper on the application of RVP during surgery on complex UIAs.

The first half of their editorial correctly summarizes the basic principles of RVP during cardiac electrophysiology and invasive cardiology procedures, e.g., transaortic valve implantation or balloon aortic valvuloplasty as a routine method in modern cardiology. ${ }^{13}$ They also stress the safety of RVP during these procedures. However, data in "cardiac healthy" patients such as in our study are scarce. Within this context it may be of interest to the readers of the Journal of Neurosurgery that RVP was first used by British neurosurgeons during surgery for complex ruptured aneurysms. The first report on RVP, at that time named "elective circulatory arrest by artificial pacemaker," was published in 1966 by Small et al. in The Lancet. ${ }^{12}$ This was followed by 4 subsequent publications between 1967 and $1971^{1,4,5,10}$ in which 75 patients were reported on for the treatment of cerebrovascular disorders (aneurysms and arteriovenous malformations), with an excellent effect of RVP on induced hypotension. During this period RVP was very often accompanied by hypothermia. However, due to long pacing periods of up to 8 minutes of RVP, the complication rate with ventricular fibrillation and the frequent necessity of open or closed chest reanimation was high. Maybe because of this complication or due to the accompanying hypothermia, this fascinating technique was, at least according to the literature, no longer used during vascular neurosurgical procedures and was forgotten.

Amazingly, the technique of RVP was then resurrected about 25 years later by our cardiological colleagues for the facilitation of stent graft repair, and from that time onward it made its way into the daily practice of invasive cardiology, as noted above., ${ }^{2,3}$ In neurosurgery it took 40 years (from 1971 to 2012) until the use of RVP was reported again, in a series of 12 patients reported by Saldien et al. from Antwerp, Belgium. ${ }^{11}$ Based on this report, we decided to embark on our study (described in the accompanying 
article) with an interdisciplinary concept, to first prospectively evaluate safety, followed by efficacy, with the final goal being to define the relevant parameters of RVP use.

As mentioned by Verma and colleagues in their editorial, under defined pre- and intraoperative precautions, including a specific cardiological workup before surgical planning and using an experienced anesthesiology and neurosurgery team, RVP is a safe procedure in aneurysm surgery. However, it should not be used casually, but only following a rigorous selection process. This process is outlined by our data, in which only 20 of 174 patients with UIAs were selected for RVP treatment during the study period. The main question, to which Verma et al. alluded to in the second half of their editorial, concerns the current and possible future role of RVP in the surgical treatment of complex UIAs.

Thus, within the context of advanced techniques for intraoperative cerebral blood flow (CBF) manipulation, we see RVP positioned between temporary clip placement and adenosine application on one side and deep hypothermia and cardiac standstill on the other side. As noted by Verma et al., temporary clip placement, despite its apparent ease of application, is not without problems considering its inherent risk of ischemia, thromboembolic events, and aggravation of cerebral vasospasm, which in combination have been shown to be as high as $25 \% .{ }^{14} \mathrm{~A}$ recent publication has additionally outlined the nonnegligible possibility of perforator injury during temporary clip placement in middle cerebral artery aneurysms. ${ }^{6} \mathrm{We}$ see the application of adenosine bolus to achieve shorttime cardiac standstill almost exclusively as a rescue medication in the case of otherwise uncontrollable intraoperative aneurysm rupture, and most of the publications regarding its use are within this context. Because of the unpredictable time frame until occurrence of asystole, and especially due to the very short duration of the effect of adenosine-which brings an unpleasant feeling of haste and uncertainty during the important period of clip deployment-we do not see adenosine as the method of choice for intraoperative CBF manipulation in complex UIAs. Additionally, and not infrequently, high and multiple doses of adenosine have to be used to achieve the desired effect. We routinely perform pre- and early postoperative MRI in our patients with UIAs undergoing either surgical or endovascular treatment. ${ }^{8}$ We have seen tiny spots of subcortical infarctions in patients after multiple adenosine doses, which have possibly escaped detection in previous studies on adenosine use because postoperative MRI examinations had not been performed.

Is there still a place for deep hypothermia and cardiac standstill? We question this, as the majority of the targets of this technology (especially complex and giant aneurysms of the posterior fossa) are now being treated with a high success rate by endovascular techniques, although in a very few select cases the necessity for this major surgical endeavor may still arise. However, the requirement of technical, medical, and human resources is remarkable, and in all series, despite impressive results, the complication rate is high. ${ }^{7}$ Although we have used deep hypothermia and cardiac standstill previously in very rare cases in our institution, in this era of increasing advancement of endovascular technology (at least over the last 8 years) we no longer have found it necessary.

Lastly, Verma et al. bring forward their request for clinical studies directly comparing RVP with alternative methods of induced intraoperative hypotension. We are, of course, well aware that a prospective, randomized, controlled double-blind trial is the ultimate scientific proof of the superiority or inferiority of a specific method compared with others. Due to the enormous variability in location, size, structure, and configuration of those complex UIAs that are a possible target of RVP application, it is our contention that it will simply not be possible to collect large homogenous groups of UIAs that can be compared with each other and different methods of inducing intraoperative hypotension. Instead, we are planning to begin a multicenter study on the use of RVP in complex UIAs to demonstrate that the promising results of our initial study on RVP, which we have now extended to more than 30 patients, can be corroborated by a broader neurosurgical community. We previously were able to demonstrate that in addition to randomized controlled trials, carefully performed prospective evaluations, especially in surgical studies, can provide a strong scientific basis to implement new treatment strategies in the neurosurgical community, ${ }^{9}$ which we hope to do again with RVP for complex UIAs.

So, to revisit the title of the editorial of Verma et al., the question is probably no longer "to pace or not to pace," to allude again to Hamlet's famous words, but to pace even more safely and effectively "in the pure brain, which some suppose the soul's frail dwelling-house" (William Shakespeare, King John, Act 5, Scene 7).

\section{References}

1. Campkin TV, Dallas SH: Elective circulatory arrest in neurosurgical operations. Br J Anaesth 40:527-532, 1968

2. Cribier A, Eltchaninoff H, Tron C, Bauer F, Agatiello C, Sebagh L, et al: Early experience with percutaneous transcatheter implantation of heart valve prosthesis for the treatment of end-stage inoperable patients with calcific aortic stenosis. J Am Coll Cardiol 43:698-703, 2004

3. Daehnert I, Rotzsch C, Wiener M, Schneider P: Rapid right ventricular pacing is an alternative to adenosine in catheter interventional procedures for congenital heart disease. Heart 90:1047-1050, 2004

4. Dimant S, Piper CA, Murphy TO: Pacemaker-controlled hypotension in surgery. Surgery 62:662-669, 1967

5. Gillingham FJ: Methods of circulatory arrest. Prog Brain Res 30:367-376, 1968

6. Joshi S, Choudhari KA: Role of temporary clipping in perforator damage during middle cerebral aneurysm surgery. $\mathbf{B r} \mathbf{J}$ Neurosurg 26:127-129, 2012 (Letter)

7. Lawton MT, Raudzens PA, Zabramski JM, Spetzler RF: Hypothermic circulatory arrest in neurovascular surgery: evolving indications and predictors of patient outcome. Neurosurgery 43:10-20, 1998

8. Platz J, Wagner M, Güresir E, You S, Konczalla J, de Rochemont R du M, et al: Early diffusion-weighted MRI lesions after treatment of unruptured intracranial aneurysms: a prospective study. J Neurosurg 126:1070-1078, 2017

9. Raabe A, Nakaji P, Beck J, Kim LJ, Hsu FPK, Kamerman JD, et al: Prospective evaluation of surgical microscopeintegrated intraoperative near-infrared indocyanine green videoangiography during aneurysm surgery. J Neurosurg 103:982-999, 2005 
10. Rovit RL: Operative hypotension for intracranial vascular surgery using pacemaker-induced ventricular tachycardia. J Neurosurg 35:51-58, 1971

11. Saldien V, Menovsky T, Rommens M, Van der Steen G, Van Loock K, Vermeersch G, et al: Rapid ventricular pacing for flow arrest during cerebrovascular surgery: revival of an old concept. Neurosurgery 70 (2 Suppl Operative):270-275, 2012

12. Small JM, Stephenson SC, Campkin T V, Davison PH, Mc-
Ilveen JS: Elective circulatory arrest by artificial pacemaker. Lancet 1:570-572, 1966

13. Webb JG, Pasupati S, Achtem L, Thompson CR: Rapid pacing to facilitate transcatheter prosthetic heart valve implantation. Catheter Cardiovasc Interv 68:199-204, 2006

14. Woertgen C, Rothoerl RD, Albert R, Schebesch KM, Ullrich OW: Effects of temporary clipping during aneurysm surgery. Neurol Res 30:542-546, 2008 